[0212-7199 (2005) 22: 6; pp 293-297] ANALES DE MEDICINA INTERNA Copyright (C) 2005 ARAN EDICIONES, S.L.

An. Med. INTERNA (Madrid) Vol. 22, N. ${ }^{\circ}$ 6, pp. 293-297, 2005

\title{
Miguel de Cervantes: saberes médicos, enfermedades y muerte
}

\author{
J. MONTES-SANTIAGO
}

Servicio de Medicina Interna. Hospital Meixoeiro. Complejo Hospitalario Uiversitario. Vigo. Pontevedra

\author{
MIGUEL DE CERVANTES: MEDICAL KNOWLEDGES, AILMENTS \\ AND DEATH
}

\section{RESUMEN}

Existe un reconocimiento generalizado de los amplios conocimientos médicos que Cervantes poseía para su época, hasta el punto de haberse afirmado que pudo ser médico. Parte de tales saberes pudieron serle trasmitidos por su padre que era cirujano-barbero y que le legó varios libros sobre materias médicas. Sin embargo, existe un desconocimiento casi total respecto a su patobiografía y las causas que originaron su muerte. Además de una posible malaria, tomando como base su propio testimonio y aceptando como síntomas cardinales la hidropesía y la sed incoercible, se le ha diagnosticado de cirrosis hepática con desarrollo final de una diabetes mellitus. No obstante, son también posibles otras alternativas como una insuficiencia cardiaca y existen ciertos argumentos a favor de que su dolencia final pudo ser una insuficiencia renal terminal.

PALABRAS CLAVE: Miguel de Cervantes. Enfermedades. Muerte.

\begin{abstract}
There is no doubt about the extensive medical knowledge of Cervantes at his time and some biographers affirm that he was a physician. Probably, part of these knowledges were the legacy of his father, a barber and surgeon, that bequeathed to him several medical books. However, there is an almost absolute ignorance related to his ailments and the cause of his death. Apart from a possible malaria, some authors have diagnosed him liver cirrhosis and diabetes mellitus, taking in account the Cervantes's own testimony, with hydropsy and uncontrollable thirst as importants findings. However, some others explanations like heart failure are possible and certain data suggest terminal renal failure as his last illness.
\end{abstract}

KEY WORDS: Miguel de Cervantes. Diseases. Death.

Montes-Santiago J. Miguel de Cervantes: saberes médicos, enfermedades y muerte. An Med Interna (Madrid) 2005; 22: $293-297$.

\section{INTRODUCCIÓN}

Con motivo del $4^{\circ}$ Centenario de la publicación de El Quijote (1605) es queja generalizada entre muchos de los estudiosos de Cervantes las notables lagunas existentes en puntos biográficos fundamentales $(1,2)$. En lo referente a sus posibles enfermedades y causas de su muerte, las lagunas se convierten en oscuridad casi total, aun a pesar de notables intentos de clarificación en el último lustro. En este trabajo se intenta revisar de forma crítica el estado de la cuestión sobre la muerte y los padecimientos del autor del Quijote.

\section{BREVE BIOGRAFÍA $(1,3-7)$}

Cervantes nació en Alcalá de Henares en 1547, conociéndose pocos datos de su infancia y juventud. Debido a las deu- das contraídas por su padre su infancia fue bastante nómada por ciudades como Alcalá, Valladolid, Córdoba, Sevilla y Madrid. La primera noticia cierta se produce en la última ciudad donde estudió con López de Hoyos y comienza a escribir sus primeros sonetos. Luego, por motivos oscuros -al parecer en relación con una refriega en la que resultó herido un hombre-, pasa a Italia, alistándose en el ejército, hasta pelear en Lepanto (1571) donde es herido. Tras larga convalecencia vuelve a enrolarse en los Tercios y cuando regresaba a casa junto con su hermano Rodrigo, es apresado por piratas berberiscos, permaneciendo cinco largos años cautivo en Argel. Aquí, dando muestras de heroísmo singular, planea prácticamente cada año una fuga, que acaban frustrándose. Una vez liberado por frailes trinitarios, que produce la ruina familiar para reunir su rescate, vive una vida de nomadeo al servicio de la Corona, que le ocasiona varios encarcelamientos y excomuniones. A partir de 1585 comienza a publicar La Galatea y

Trabajo aceptado: 17 de junio de 2005

Correspondencia: Julio Montes Santiago. Hospital Meixoeiro-CHUVI. 36200. Vigo. e-mail: julio.montes.santiago@sergas.es. Tlfno. 9868111 (ext. 541). 
piezas teatrales que le proporcionan cierto reconocimiento. No será, sin embargo hasta la publicación de la primera parte de El Quijote (1605), cuando comience a ser conocido y frecuentar los círculos literarios. La irrupción de Lope Vega en el panorama teatral arruina sus esperanzas en este campo, pero sin embargo, cumplidos ya los 65 años y en un periodo final prodigioso (1613-1616), publica sucesivamente las Novelas Ejemplares, El Viaje al Parnaso, la segunda parte del Quijote, Las Comedias y Entremeses, el Persiles y Segismun$d a$, y deja inacabadas varias obras.

\section{LOS SABERES MÉDICOS DE CERVANTES}

1. Los familiares médicos $(7,8)$. Desde que Américo Castro sugiriera el posible origen judeoconverso de la familia de Cervantes, ríos de tinta se han vertido sobre este tema (7). El apasionamiento ha sido importante, y, mientras ilustres cervantistas como Astrana Marín niegan dicho origen (3), otros no menos ilustres, lo defienden con entusiasmo. Sin entrar en el fondo de la polémica, el motivo de traerla a colación es que uno de los principales motivos alegados para afirmar dicho origen judeoconverso, es la profesión de cirujano-barbero de su padre Rodrigo, así como la de su bisabuelo materno. En efecto, su abuelo Juan Cervantes se casó con Leonor, hija del bachiller médico y cirujano Juan Díaz de Torreblanca. En aquellos tiempos la profesión de médico y barbero era mayoritariamente ejercida por personas de ascendencia judía, afirmándose que 9 de cada 10 médicos eran de dicha ascendencia (8), entre ellos los famosos Andrés Laguna o Rodríguez de Villalobos.

Rodrigo, que era sordo y que quedó en una difícil situación cargado de hijos cuando su padre Juan, de acomodada posición, abandona a su mujer y a sus hijos en Alcalá para irse a Córdoba, era cirujano barbero y ejercía en el Hospital de Antezana (fundado en 1483). Aunque en España la regulación de los cirujanos-barberos era algo diferente al resto de Europa (8), el estamento de estos no era muy elevado como muy bien señala el propio Cervantes en el Entremés El Juez de los Divorcios. En él distingue claramente a los "médicos del pulso", de nivel y conocimientos más elevados, de los cirujanos barberos y sangradores, de condición mucho más humilde. No obstante, Rodrigo, al parecer, aunque sin excesiva suerte, era aficionado a las lecturas, legando a su hijo varios volúmenes. Aunque no sabemos los conocimientos exactos de Rodrigo, examinando las fechas de su estancia en Alcalá es preciso comentar la interesantísima situación vivida entonces en aquella universidad, y señalada por López Piñero (9-11). En efecto, Miguel había nacido en 1547, pero debido a las deudas y a la denuncia del Marqués de Cogolludo al padre de Miguel por la incapacidad de curar a un hijo suyo, toda la familia deben emigrar a Valladolid en 1551, regresando a Alcalá en 1553 para casi inmediatamente partir Rodrigo para Córdoba (6). En aquellos tiempos había estallado la polémica en España sobre la nueva visión anatómica producto de la publicación del libro Humanis Corporis Fabrica (1543), de Andrea Vesalio. Hasta ese momento la disección de cadáveres estaba en manos de prácticos como los cirujanos barberos. El profesor iba indicando a un ayudante la forma de la disección, quien a su vez se la comunicaba al cirujano. Con Vesalio esto cambia y es el propio médico quien realiza las disecciones, tal como puede observarse en la primera lámina de la Fabrica. En este momento Vesalio está en España como médico de Cámara de Carlos V, aunque no consta que se dedicara a la enseñanza académica. Sin embargo, los principales valedores de sus enseñanzas eran sus discípulos Jimeno y Collado, que enseñaban en la Universidad de Valencia. Pero, precisamente el primero se había trasladado a Alcalá en 1550 ó 1551. Aunque fueran ellos mismos los que practicaban las disecciones, ¿acudió el inquieto Rodrigo a sus clases?. Se desconoce. Sí consta, sin embargo, que sus familiares pretendieron que había alcanzado el grado de bachiller en Medicina, aunque esto no era cierto.

2. La biblioteca médica de Cervantes. Estudios recientes, fundamentalmente de Eisenberg, parecen desmontar uno de los más persistentes mitos cervantianos: su extrema pobreza (12). No cabe duda de que pasó necesidad en ciertas épocas y hubo de ganarse la vida desempeñando oficios de intendencia mal pagados, que le ocasionaron numerosos sinsabores, y en uno de los cuales -quizá su encarcelamiento en Sevilla en 1589- pudo nacer el Quijote "engendrado en una prisión“ (I, Prólogo). Sin embargo, parece haber reunido una importante biblioteca -Eisenberg contabiliza hasta 214 libros (12)- con el dinero percibido por sus piezas teatrales. Estas, como $L a$ Numancia y otras obras hoy perdidas, le habrían reportado una cierta notoriedad, a diferencia de La Galatea que pasó desapercibida. Pues bien, entre sus libros había algunos de tema casi exclusivamente médicos. Algunos de ellos -Practica in Arte Chirugica Copiosa de Giovanni Vigo (Traducción de Miguel Juan Pascual, 1537), el Tratado de las cuatro enfermedades cortesanas de Lobera de Ávila (1544), la Practica y Theorica de Cirugia (1582) de Daza Chacón, el Dioscórides comentado e ilustrado por Andrés Laguna (1555), mencionado en el Quijote (I,18)- proceden probablemente del legado de su padre Rodrigo, muerto en 158512. El Tratado de todas las enfermedades de los riñones...de Francisco Díaz (1588), probable amigo de su padre desde los tiempos de Alcalá, fue seguramente regalo del autor como agradecimiento al Soneto escrito por Cervantes en su Prólogo y a la inclusión del médico entre los autores de El Viaje al Parnaso. Otro libro famoso de su biblioteca fue seguramente El Examen de Ingenios de Juan Huarte de San Juan, publicado en 1575, incluido en el Índice de Libros prohibidos y retirado en 1583 y reeditado con correcciones en 1594. Es evidente que además de tomar prestado el título de Ingenioso Hidalgo para su D. Quijote, Cervantes utilizó muchas de las ideas de Huarte, p.ej el carácter nervioso de D. Quijote o el flemático de Sancho, etc.-, e incluso copió literalmente varios pasajes del mismo (13).

3. Sus conocimientos médicos y su repercusión ulterior en la Medicina. Es notorio que Cervantes poseía amplios conocimientos médicos para la época y El Quijote es buen ejemplo de ello, como muestran recientes publicaciones (14), entre las que es de consulta obligada el exhaustivo estudio La Antropología Médica y El Quijote, del profesor de Medicina Forense JM Reverte Coma, recientemente actualizada y puesta a disposición general en Internet (15). Es significativo mencionar en este terreno que la constatación de tales conocimientos llevaron a afirmar a alguno de sus estudiosos que el propio Cervantes había sido médico (16).

Sobre sus repercusiones ulteriores y siguiendo la advertencia de Unamuno resistiremos la tentación de aventurar hipótesis sobre las dolencias del Ingenioso Hidalgo. Decía el rector de Salamanca que los médicos eran los peores comentaristas 
de Don Quijote porque todo lo liaban con sus términos técni$\cos (17)$. Aunque parece ser que para el Licenciado Vidriera Cervantes tomó ejemplo de algún caso real (18), aquí se obviarán los repetidos intentos por llevar a D. Alonso Quijano al diván. No obstante, si se trata de recordar la naturaleza de su enfermedad mental, es preciso señalar que el mismo Sigmund Freud aprendió español en sus años jóvenes para poder leer El Quijote, y son muy evidentes sus influencias p.ej. en su Tratado de los Sueños (19). En la estela de Freud, otro personaje señero en la Historia de la Neurología, Wilder G. Penfield, amigo de Osler y Sherrington, descubridor junto con Rasmussen de los homúnculos sensitivo y motor en la corteza cerebral, que viajó a España en 1924 para estudiar junto a Del Río-Hortega y Cajal, dejó resumida su estancia y admiración con estas palabras: "Aprendimos a amar la tierra de Don Quijote. Allí encontramos el oro de la ciencia y el tesoro del arte. Sí, Rocinante, nos había servido bien" (20). Y no puede dejar de recordarse la conocida anécdota del "Hipócrates inglés" Thomas Sydenham (1624-1689), -creador del concepto de las enfermedades como especies nosológicas-, quien preguntado por su discípulo Richard Blackmore acerca de un modelo para estudiar medicina, respondió "lee El Quijote"(21).

\section{ENFERMEDADES Y MUERTE DE CERVANTES}

Como queda apuntado son pocos los datos conocidos sobre los padecimientos de Cervantes. Una revisión de los mismos sería la siguiente:

a) El cuadro febril en la víspera de la Batalla de Lepanto. Este motiva que sus superiores decidan dejarlo sin combatir, decisión que sólo la obstinación de Cervantes consigue revocar. El análisis de los cuadros febriles en los hospitales del siglo XVII indican que estos eran la causa más frecuente de ingreso y en su mayoría eran causados por malaria (fiebres tercianas y cuartanas) (22). Dada la endemia de malaria en Italia y Roma -recuérdese p.ej. que tres siglos después el pintor Mariano Fortuny fallecerá cerca de Roma a causa de ella-, no es aventurado suponer que Cervantes pudo contraerla durante su estancia en Italia y algunos autores afirman que la contrajo en 1569, dos años antes de su participación en Lepanto (20). Aquí recibió dos heridas en el pecho y una en la mano izquierda, que le dejaron sin su uso. Dichas lesiones debieron ser graves o las complicaciones importantes, puesto que Cervantes tardó en recuperarse siete meses en un hospital de Mesina. No obstante, una vez repuesto vuelve a enrolarse en la Armada, participando en acciones bélicas como Navarino. Y no cabe duda de que Cervantes conocía bien la malaria. Aparte de otras referencias, bastaría sólo señalar el paralelismo entre los síntomas que anteceden a la muerte de Don Quijote (II,74) y la descripción clínica de la malaria que se realiza en el más prestigioso Tratado de Enfermedades Infecciosas (23) -con sus periodos de altísima fiebre, de intenso sueño y recuperación del episodio-, para demostrar que, o bien Cervantes la padeció o bien la presenció muy de cerca.

b) Los achaques del final de la vida. Aparte de una dolencia poco definida en 1592 (4), probablemente debida a las penurias ocasionadas por el retraso en el pago de las cantidades adeudadas como recaudador de la Corona, el siguiente síntoma es la queja sobre el cansancio que aparece en el Prólogo de las Novelas Ejemplares (1613). Dicha queja es demasiado inespecífica. Cervantes tiene en este momento 66 años, edad que puede calificarse de singularmente avanzada para aquella época, en la cual la misma vejez era por sí misma motivo de ingreso hospitalario. Y se cita como verdaderamente excepcional el caso del médico Luis Mercado que alcanzó la edad de 79 años (22).

c) La hidropesía. Prácticamente la totalidad de los diagnósticos a la muerte de Cervantes se han basado en lo escrito o dictado por éste en el Prólogo de su obra póstuma Los Trabajos de Persiles y Segismunda. En él Cervantes describe literariamente que mientras se dirige a Toledo desde Esquivias, se encuentra con un estudiante que al reconocerlo le manifiesta su regocijo. Cervantes le invita a hacer juntos el camino y la conversación recae sobre su enfermedad. Entonces el estudiante le dice que no abandone su alimentación y que su enfermedad es hidropesía, que da una sed terrible, que no es aliviada aunque se bebiera todo el agua del océano. Cervantes asiente diciendo que eso también le han dicho muchos, pero que no puede dejar de beber. En estos dos datos -hidropesía y sed intensa- se han basado los diagnósticos actuales sobre la enfermedad de Cervantes, que intentan superar los muy vagos de "arteriosclerosis", muy de moda a principios del siglo XX (24). Uno con cierto predicamento, aceptado por ilustres cervantistas médicos y no médicos, aunque en ciertos casos con ausencia de crítica, es el de cirrosis hepática con desarrollo final de una diabetes mellitus $(6,7,25-27)$. A esta hipótesis se ha contrapuesto alternativamente la de una insuficiencia cardiaca de posible origen hipertensivo $(18,21)$.

Para centrar adecuadamente el problema es preciso señalar las siguientes cuestiones: A) Según se ha señalado, el prólogo del Persiles, como casi todos los otros de Cervantes, es un prólogo literario y que como tal puede ser real, inventado o una mezcla de ambos (5). B) Cervantes empleó dos veces el término "hidrópico" -en El Quijote y en El Viaje al Parnaso-, aparentemente con el significado de ascitis (6). No obstante, en el Tesoro de la Lengua Castellana o Española de Sebastián de Covarrubias publicado en 1611, por tanto entre la $1^{\mathrm{a}}$ y $2^{\mathrm{a}}$ Parte del Quijote, "hidropesía" hace referencia a la hinchazón generalizada de los tejidos, tanto del abdomen como de las piernas, y vendría a asimilarse al término de anasarca (21). Si se considera esta acepción las tres entidades más frecuentes que producen edemas generalizados serían la cirrosis hepática, una nefropatía con posible síndrome nefrótico acompañante o la insuficiencia cardiaca. Respecto a la última mencionada, se hace difícil concebir que Cervantes omitiera en el viaje referido síntomas tan evidentes como la ortopnea o disnea de mínimos esfuerzos que podría producirle habla entrecortada. Del relato parece desprenderse que su charla con el estudiante fue animada y no apareció tan característico dato. C) Se ha aducido la sed incontenible como síntoma muy sugestivo de una diabetes mellitus. Tal trastorno, aunque conocido desde las descripciones clásicas de Areteo de Capadocia (S. I dC) sólo adquirirá rango de plena entidad en 1790 con la diferenciación entre diabetes mellitus e insípida realizada por Johann Peter Frank. Se ha argumentado que la diabetes y la sed podrían ser eventos finales en una cirrosis hepática. Sin embargo, tal interpretación olvida mencionar una cuestión importante. El Tesoro de Covarrubias recoge para "hidropesía", junto a edemas generalizados, la acepción de avaricia pues se consideraba que todos los hidrópicos padecían una sed incoercible similar a la necesidad incontenible de los avaros de acumular riquezas (21). De hecho esta significación, perdiendo parte de su connotación médica, pasa a ser predominante en el lengua- 
je literario. Con este sentido de sed o deseo intenso existen textos muy significativos, p. ej. de Quevedo, contemporáneo de Cervantes, quien incluso llegar a inventar palabras como "libropesía" o sed insaciable de libros, en las que juega con este significado (28). Además, aunque en las cirrosis puede desarrollarse diabetes, en otros casos avanzados se observa hipoglucemia.

Un problema adicional, semejante al que se presenta en los estudios retrospectivos y fuente de grandes errores, es el siguiente: ¿el que un signo o síntoma no aparezca reflejado denota que está ausente o que fue omitido? Esto es particularmente relevante en el caso de Cervantes, porque no sabemos si existió el otro gran signo acompañante de la diabetes: la poliuria. Es improbable que este síntoma fuera desconocido de Cervantes, que había escrito el prólogo al libro urológico de Francisco Díaz, o que se refiere a la urgencia miccional de uno de los galeotes del Quijote (I, 22). Por tanto, si lo omitió conscientemente el diagnóstico de diabetes sigue siendo posible, pero si no existió entonces caben hipótesis alternativas, entre ellas que la hidropesía estuviera en relación con una nefropatía terminal que cursara con oligoanuria y es clásico el síntoma de sed incoercible de la uremia (29). Si la hipótesis aventurada es cierta, es decir, que Cervantes pudo padecer una nefropatía con desarrollo de síndrome nefrótico y ascitis, que terminó con su vida, ¿habría alguna orientación etiológica?. Ya se ha mencionado que se ha aventurado el diagnóstico de insuficiencia cardiaca -¿también renal?- de presumible origen hipertensivo. Aunque si admitimos que Cervantes padeció malaria en su juventud, ¿desempeñaría cierto papel etiológico?. Ha sido descrita una glomerulopatía que puede cursar con síndrome nefrótico por depósito de inmunocomplejos IgG, IgM C y Ag. de plasmodium-, con posible evolución hacia una uremia terminal (30).

d) Otros datos. ¿Hay alguna otra noticia en los escritos cervantinos que pueda ayudar a emitir alguna hipótesis sobre su enfermedad? Quizá pueda ser relevante un dato negativo. Cuando hace su autorretrato literario en el Prólogo de las Novelas Ejemplares se describe como de tez más pálida que morena. Esto, evidentemente, no descarta nada de manera definitiva, aunque en el caso de una hepatopatía avanzada podría ser ya evidente cierta ictericia que en esta descripción no aparece. En la etiología de esta posible hepatopatía -una causa podría otra vez ser la malaria- puede descartarse de for- ma casi segura el alcoholismo. No hay ningún dato ni en Cervantes ni en sus contemporáneos que indique que fuera bebedor excesivo (25). Ello es significativo, puesto que ni en las más furibundas invectivas de sus detractores como Avellaneda, o en ocasiones Lope de Vega, se le acusa de ello. Asimismo, sabemos por el testimonio del último que Cervantes utilizaba anteojos, pero esto bien podría ser más derivado de la edad que de otra patología. Desde luego, si Cervantes padeció diabetes, la posible retinopatía no parece haber sido importante, dada su ingente producción en los últimos tres años. En el reverso de la moneda quizá merezca mencionarse que Gregorio Marañón en su libro Los estados prediabéticos (1927) ya consideraba la piorrea como tal estado, como ha hecho notar recientemente Serrano Ríos (II Simposium Nacional de Síndrome metabólico, marzo 2005). Pues bien, tal concepto en forma de fuerte asociación entre enfermedad periodontal y diabetes ha sido recientemente rescatado (31). Naturalmente, puede haber otras explicaciones pero, ¿pudo la diabetes ser causa de los "dientes ni menudos ni crecidos, porque no tiene sino seis, y esos mal acondicionados" de su autodescripción en el Prólogo mencionado?

Poco sabemos, si hacemos caso al Prólogo del Persiles, de los muchos a los que habría consultado sus dolencias Cervantes. Sus amigos médicos, como el Doctor Rodríguez de la Fuente, al que menciona en La Ilustre Fregona o Francisco Díaz habían muerto ya. Alonso López Pinciano, médico vallisoletano, que actuó como revisor del Quijote, estaría preferentemente dedicado a las tareas literarias. Por último, conviene ser precavido sobre ciertos datos biográficos. Es significativo que la carta supuestamente dirigida por Cervantes al Cardenal Sandoval y Rojas en la que se declara muy enfermo, que durante más de un siglo presidió las sesiones de la Real Academia Española de la Lengua, resultara ser un fraude, como también el famoso retrato realizado por Juan de Jáuregui, que seguramente es sólo una falsificación hecha por un tal José Albiol a principios del siglo XX $(2,5)$.

En conclusión, siguen persistiendo numerosas lagunas sobre las causas definitivas de la enfermedad mortal de Cervantes. A los diagnósticos emitidos de cirrosis hepática con desarrollo de una diabetes mellitus, y al de insuficiencia cardiaca, quizá debería añadirse la hipótesis, que cuenta con algunos argumentos a favor, de una uremia terminal con desarrollo de un síndrome nefrótico y anasarca como causa de su muerte.

\section{Bibliografía}

1. Martín de Riquer. Biografía de Cervantes. En: Miguel de Cervantes, Don Quijote de la Mancha. Edición revisada, introducción y notas de Martín de Riquer. Planeta: Barcelona, 2004; p. XXVIII-XXXI.

2. Eisenberg D. Los trabajos del biógrafo cervantino. Bull Cervantes Society America 2003; 23.1:235-49.

3. Astrana Marín L. Vida ejemplar y heroica de Miguel de Cervantes Saavedra.. 7 vols. Madrid: Reus, 1948-58.

4. Emerson PS. Index of Astrana Marín's Vida ejemplar y heroica de Miguel de Cervantes Saavedra, with a Chronology of Cervantes' Life. Cronología de eventos en la vida de Miguel de Cervantes Saavedra, según su biógrafo, Luis Astrana Marín. [consultado 05-02-2205]: disponible en: http://www.h-net.org/ cervantes/csa/emerson_index.htm.

5. Canavaggio J. Cervantes. Madrid: Espasa Calpe (Colección Austral), 2003.
6. Vidal C. Enciclopedia del Quijote. Barcelona: Planeta, 1999.

7. Alvar Ezquerra A. Cervantes. Genio y libertad. Madrid: Temas de Hoy, 2004.

8. Gómez-Menor Fuentes J. La oscura genealogía de Cervantes. [consultado 0502-2005]: disponibleen:_http://biblioteca2.uclm.es/biblioteca/ceclm/artrevistas/Toletum/tol33/toletum33_gomezoscura.pdf).

9. López Piñero JM. Tradición y renovación en la medicina española del renacimiento. En Viejo y nuevo continente: La medicina en el encuentro de dos mundos. López Piñero, coordinador. Madrid: Saned, 1992, p. 34-88.

10. López Piñero JM. El Renacimiento. En: La Medicina en la Historia. Madrid: La esfera de los Libros, 2002, p. 173-284.

11. López Piñero JM. I saperi morfologi e l'illustrazione anatomica. (Traduzione de Elena Melchiorri). En: Rippa Bonati M y Pardo-Tomás J, editores. Il Teatro dei Corpi. Le Pitture colorate d'anatomía di Girolamo 
Fabrizi d'Acquapendente. Venecia: Médiamed Edizione Scientifiche, 2004, p. 49-62.

12. Eisenberg D. La biblioteca de Cervantes. Una reconstrucción (2002). [consultado 05-02-2005]: disponible en http://bigfoot.com/ daniel. eisenberg)

13. Martín-Araguz A, Bustamante-Martínez C. Examen de ingenios, de Juan Huarte de San Juan, y los albores de la Neurobiología de la inteligencia en el Renacimiento español. Rev Neurol 2004; 38:1176-85.

14. Reverte Coma JM. La Antropología Médica y el Quijote. [consultado 05-02-2005]: disponible en http://www.ucm.es/info/museoafc/home. html.

15. Torres B. Cuerpo y gesto en el Quijote de Cervantes. Biblioteca de estudios Cervantinos: Madrid, 2002, p. 147-280.

16. Villechauvaix J. Cervantes, malade et médecin (1898). Citado en Simini B. Miguel de Cervantes, hydropsy, and Thomas Sydenham. BMJ 2001; 323:1293.

17. Pérez N. Don Quixote's diagnoses: an historical approach. 12/11/2003. [consultado 07-03-2005]: disponible en: http://www.fictionethics.org/ aps/Paper/11.

18. Bailón Blancas JM. Cervantes y la Psiquiatría. La histeria en El Licenciado Vidriera. (Reseña de Sainz SG). Diario Médico, 18/07/2000. [consultado 02-02-2005]: disponible en http://www.diariomedico. com/entorno/ent 180700 com.html.

19. Grinberg L, Rodriguez JF The influence of Cervantes on the future creator of psychoanalysis. Int J Psychoanal. 1984; 65 (Pt 2):155-68.

20. García-Albea E. Wilder G. Penfield en la residencia de estudiantes (Madrid, 1924). Rev Neurol 2004; 39:872-8.

21. Simini B. Miguel de Cervantes, hydropsy, and Thomas Sydenham. BMJ 2001; 323:1293.
22. Rojo Vega A. Enfermedades hospitalarias en la España interior del Siglo XVII. Medicina \& Historia 2000; (4 época) 1: 1-16.

23. Krogstadt DJ. Plasmodium species (Malaria). In: Mandell GL, Bennett JE, Dolin R, editors. Principles and Practice of Infectious Dseases. 5th ed. Philadelphia: Churchill Lvingstone. 2000; p. 2817-31.

24. Fitzmaurice-Kelly J. Miguel de Cervantes Saavedra; reseña documentada de su vida (Oxford, 1917). [consultado 05-02-2005]: Disponible en http://www.proyectoquijote.com.

25. López Alonso A. Enfermedad y muerte de Cervantes. Madrid: Editorial Universidad Alcalá de Henares, 1999.

26. Gil Extremera B. La diabetes terminal de Miguel de Cervantes. En: Genio y figura. Enfermedad, historia y proceso creador. Madrid: Doyma, 2002; p.29-31.

27. Pérez Miranda M. Pacientes ilustres de la historia. [consultado 15-12-2003]: disponible en http://www.unex.es/sric/archivos/abril04/pacientes.pdf.

28. Alarcos García E. Quevedo y la parodia idiomática. [consultado 05-022005]: disponible en http://cvc.cervantes.es/obref/satiras_quevedo/alarcos.htm.

29. Denker BM, Brenner BM. Azotemia and urinary abnormalities. In: Kasper DL, Fauci AS, Longo DL, Braunwald E, Hauser SL, Jameson JL, editors. Harrison's Principles of Internal Medicine. 16th ed. New York: McGraw-Hill, 2005; p. 246-52.

30. Chugh KS, Sakhuja V. Glomerular disease in the tropics. In: Davison AM, Cameron JS, Grünfeld J-P, Kerr DNS, Eberhard R, Winearls CG, editors. Oxford Textbook of Clinical Nephrology. 2th ed. Oxford: Oxford University Press, 1998; p. 703-19.

31. Saremi A, Nelson RG, Tulloch-Reid M, Hanson RL, Sievers ML, Taylor GW et al. Periodontal disease ane mortality in type 2 diabetes. Diabetes Care 2005; 28: 27-32. 